\title{
VIII. Remarks on a simplification of the theory of vibratory motions
}

\section{Cellérier}

To cite this article: C. Cellérier (1880) VIII. Remarks on a simplification of the theory of vibratory motions, Philosophical Magazine Series 5, 10:59, 57-60, DOI: 10.1080/14786448008626884

To link to this article: http://dx.doi.org/10.1080/14786448008626884

曲 Published online: 28 Apr 2009.

Submit your article to this journal

Џ Article views: 2

Q View related articles $\square$ 
build further conclusions on the existence of such selective reflection. I will merely point out that if the reflecting-power of incandescent gases for certain groups of rays is considerably greater than for all others, this will not be without importance for the spectroscopic investigation of heavenly bodies which, like comets for instance, emit partly their own, partly reflected light. If the light of such a body consists of single isolated groups or shows narrower brighter bands on a continuous dark spectrum, we are accustomed, according to our present knowledge, to assume that the light of this discontinuous spectrum is entirely and exclusively emitted by the body as a selfluminous one. If the body possesses a selective reflectingpower, the above conclusion is not at once admissible. One might even imagine, as the most extreme case, a non-luminous very dense mass of gas in our solar system, possessing selective absorption, and consequently for many separate groups of rays a power of selective reflection. Such a mass, intensely illuminated by the sun, would, without being self-luminous, show a discontinuous spectrum by reflection.

Strassburg, March 1880.

\section{Remarks on a Simplification of the Theory of Vibratory Motions. $B y$ C. Cellérier*.}

TWE motions in question are the oscillations of the particles 1 on both sides of their positions of equilibrium-that is to say, those which constitute sound and light. To find their law on the most general hypothesis, the excursions and the molecular velocities at a fixed instant called the initial instant are supposed to be given; the unknowns are the projections of those excursions upon three rectangular axes at any instant whatever: they are functions of the time and of the position of the particle.

The equations of the motion are satisfied by taking for each of them a sum of terms of the form $a \cos \rho(p-s t)$, in which $t$ is the time, $p$ the distance of the particle from a fixed plane, and $a, \rho, s$ constants. The motion represented by one of these terms isolated is called a simple motion.

At the initial instant each of the terms reduces to $a \cos p p$; and the constants and the fixed plane corresponding to each can be arranged so that their sum shall reproduce the initial

* Translated from the Archives des sciences Physiques et Naturelles of the Bibliothèque Universelle, June 5, 1880, pp. 549-553, having been communicated to the Sociéte de Physique et d'Histoire naturelle of Geneva on June $3,1880$. 
values of the excursion-that is to say, any given function $f$ of the position of the point in space. For this we must have

$$
f=\Sigma a \cos \rho p \text {. }
$$

This mode of representation of a function is Fourier's formula.

The values of the unknowns thus found are sextuple integrals, an exact solution of the problem, but which give no idea of the general form of the motion. It is only after laborious transformations, supposing the initial disturbance included within a limited space, that we arrive at interpreting them so as to make evident the limited wave-form.

Beside this complication, Fourier's formula has another inconvenience: the integration with respect to some of the variables has, if we commence with them, an indeterminate result; and differentiation under the symbol of integration offers bat little guarantee of accuracy.

Now these inconveniences may be avoided by substituting for Fourier's formula another, likewise representing an arbitrary function $f(x, y, z)$ of three indeterminates which may be regarded as rectangular coordinates of a variable point: the function is supposed $=0$ if the point is outside of a limited space designated by V. For the enunciation of the formula, we will denote by $\mathrm{S}$ a spherical surface having nnity for radius, and for its centre the origin $\mathrm{O}$; it shall be divided into elements $\omega$; designating by $\mathrm{H}$ the position of any one of them, $\alpha, \beta, \gamma$ will be the cosines of the angles made by the straight line $\mathrm{OH}$ with the axes; $\mathrm{P}$ will denote a plane perpendicular to $\mathrm{OH}$, cutting this straight line at a distance $p$ from the origin, which distance will be taken as negative on the side opposite to $\mathrm{H}$; lastly, we will put

$$
\mathrm{F}(p)=\Sigma f^{\prime}\left(x^{\prime}, y^{\prime}, z^{\prime}\right) \omega^{\prime},
$$

the summation extending to all the elements $\omega^{\prime}$ of the plane $P$, and $x^{\prime}, y^{\prime}, z^{\prime}$ being the coordinates of each.

Thus $\mathrm{F}(p)$ will be a function solely of $p$ and the cosines $\alpha, \beta, \gamma$. Let $\phi(p)$ be its second derivate with respect to $p$, in taking which $\alpha, \beta, \gamma$ are regarded as constants. The following is the formula sought:-

$$
f(x, y, z)=-\frac{1}{8 \pi^{2}} \Sigma(\alpha x+\beta y+\gamma z) \omega,
$$

in which the symbol of summation extends to all the elements $\omega$ of the sphere $\mathrm{S} ; a, \beta, \gamma$ correspond to each; $x, y, z$ are the coordinates of any point in space.

The preceding expression comprises in the main four inte- 
grations, all within finite limits and without indetermination; they can be carried out rigorously; and we can thus demonstrate that $f(x, y, z)^{*}$ is found again as its value.

If we express in this way the initial functions, and reduce them all to the single function corresponding to one and the same element $\omega$, they will be found to be functions of a single coordinate $\alpha x+\beta y+\gamma z$, which is the abscissa of any point whatever, counted in a direction parallel to the fixed direction $\mathrm{OH}$; moreover they will have values different from $\mathrm{O}$ only within a restricted region : for example, the abscissa must be comprised between $\pm \mu$, attributing to the volume $\mathrm{V}$ the form of a sphere of radius $\mu$ and placing the origin at the centre.

Now, in this case the equations of the motion are integrated immediately, the excursions are functions solely of the same abscissa, and the simple motion which results is as easy to find as if it were represented by a cosine ; only it is of quite a different nature and composed of a limited plane wave. Let us call that plane which was at first carried through the origin perperdicular to $\mathrm{OH}$ the middle plane; and suppose it to be displaced parallel, with a constant velocity $\mathrm{S}$. The disturbed region will at each instant be bounded by two planes parallel to that plane, taken on both sides at the distance $\mu$.

The total motion results from the superposition of these plane waves; their middle planes form, at the end of the time $t$, the whole of the tangent planes to one and the same interior envelope, which is the surface of the waves; the motion is sensible only within a small thickness on both sides of the envelope; and it can be ascertained by a very simple reasoning that the prolongations of the plane waves outside of this limited region interfere with one another, at least at a notable distance from the centre of disturbance.

Either the complete integrals given by Poisson for an isotropic medium, or, in the case of a crystallized medium, the law of the motion for a point at a distance from the origin is also found with sufficient facility. The latter is found to depend on the radius of curvature of the apparent contour of the surface of the waves upon any plane which passes through one of its normals. Now it is remarkable that, if a secant plane parallel to the former be carried through the same normal, the product of the radius of curvature of the section into that of the contour is constant; besides, the radii of curvature of the surface of the waves are determined by those of the surface of the sixth order which represents the velocities; so that the formulæ no longer contain any thing unknown.

* For further details see the Mémoires de la Société de Physique de Genève, année 1880. 
It is moreover immaterial whether we employ in the results Fresnel's representation of the surface of the waves or that deduced from Cauchy's theorem: the latter only is theoretically accurate; but $I$ have before demonstrated that, on determining by experiment the constants which enter into it, it coincides with Fresnel's, and that with an exactness exceeding that of the observations.

It is remarkable that the transformation indicated above for Fourier's formula has no existence for a function of two variables. This fact is connected with another, viz. that there cannot exist a limited cylindrical wave, at least interiorly.

Supposing the initial disturbance circumscribed within an indefinite vertical cylinder, admitting moreover that the excursion and the velocity are at that instant the same for all points situated on one and the same vertical, this will certainly be the case during the whole motion; and this will have the form of a vertical cylindrical wave propagating itself horizontally in all directions; but the whole of the points interior to the cylinder will remain indefinitely in motion; so that it cannot be resolved into limited plane waves.

IX. On a Simple Method of Galvanic Calibration of a Wire. By V. Strouhal and C. BarUs*.

THE methods which are customarily used for the calibra1 tion of a wire (as, for example, in the Kirchhoff-Wheatstone bridge-combination) undoubtedly labour under the disadvantage that for carrying them out resistances are required beforehand which have been equalized in another way under certain simple conditions. In this way the accuracy of the desired result is made dependent on that of a previous equalization, whereby the errors to be expected in the calibre of the wire are placed in doubt by unavoidable errors in the equalization of the resistances, and so much the more the smaller they happen to be. Hence the importance of the problem for precise determinations by the otherwise so convenient bridge method constantly justifies the endeavour to get rid of this inconvenience as completely as possible, and, in addition, by the simplicity of the means propounded, to make the execution to the utmost convenient and facile.

The method described in the following, which we have repeatedly employed with advantage, is perfectly analogous to that which is usually applied, in the well-known manner, to 330.

Translated from Wiedemann's Annalen, 1880, No. 6, vol. ¿. pp. 326- 